Results From 814 age- and sex-matched controls identified, a 25\% questionnaire response rate was achieved. $3 \%$ were returned with address inaccuracy, and no complaints were received from any recipient. Of the 163 hospital attendees, 135 (83\%) were identified on the website. Comparing those who were/were not identified revealed no differences in mean age (identified $=59$ yrs, vs $60 \mathrm{yrs}$; $\mathrm{P}_{\text {Mann-Whitney }}=0.45$ ), sex $\quad$ (identified $=50 \%$ Female vs $57 \%$; $\left.\mathrm{P}_{\chi}^{2}=0.51\right)$, deprivation $\left(\mathrm{P}_{\text {Mann-Whitney }}=0.71\right)$ or rurality $\left(\mathrm{P}_{\chi}^{2}=0.11\right)$. Of those identified, an age estimate was quoted for 131 (97\%) of which $113(86 \%)$ were accurate.

Conclusions This is the first evaluation of an online population sampling frame of which we are aware. The website, 192.com, acted as a feasible sampling frame, demonstrating good participant acceptability, coverage, accuracy and representation. In the future, such resources could provide researchers with alternative sampling opportunities.

\section{P1-393 COMMUNITY BASED NOISE INDUCED HEARING LOSS PREVENTION FOR TRIBAL CHILDREN}

\section{doi:10.1136/jech.2011.142976f.84}

${ }^{1} \mathrm{~T}$ Becker, ${ }^{*}{ }^{1} \mathrm{~W}$ Martin, ${ }^{1} \mathrm{~W}$ Lambert, ${ }^{1} \mathrm{~S}$ Griest, ${ }^{2} \mathrm{~J}$ Sobel. ${ }^{1}$ Oregon Health \& Science University, Portland, Oregon, USA; ${ }^{2}$ Portland State University, Portland, Oregon, USA

Introduction The purpose of this study is to evaluate the effectiveness of a community based, multi dimensional noise induced hearing loss (NIHL) and tinnitus prevention program in American Indian elementary school children. NIHL is responsible for $30 \%$ of all hearing loss nationally and is almost $100 \%$ preventable with simple measures.

Methods Baseline questionnaires identifying knowledge, attitudes and behaviours regarding sound exposures of $4^{\text {th }}$ and $5^{\text {th }}$ grade student of an Oregon Tribal School were completed. Community intervention includes use of local media modalities, presentation of a classroom education program, and a community/family event and booster intervention with a web-based virtual museum exhibit. Post intervention and 3 month follow-up questionnaires will be collected to evaluate short and long-term changes in parameters

Results Information was provided to the community via Tribal newspaper, radio and web sources. Baseline questionnaires were completed by 130 students. 160 students received the Dangerous Decibels classroom program. 225 children, friends and family attended an evening event furthering the education. Students selfreported a wide range of hazardous sound exposures, most listening to loud music and fireworks. Over 31\% reported regular exposure to live gunfire. $18 \%$ use hearing protection frequently. $53 \%$ reported being around sounds that were loud enough to cause pain or tinnitus.

Conclusions These results indicate that the target population of the study is at risk for NIHL and related tinnitus. This is a first attempt at improving hearing health in a Tribal community through community based intervention.

\section{P1-394 HEALTHY COMMUTING: INDIVIDUAL COGNITIONS AND NEIGHBOURHOOD FACTORS ASSOCIATED WITH WALKING AND CYCLING TO WORK}

doi:10.1136/jech.2011.142976f.85

M Beenackers, ${ }^{*}$ C Kamphuis, F van Lenthe. Erasmus University Medical Center, Rotterdam, The Netherlands

Introduction Physical inactivity is among the most important and prevalent risk factors of many major diseases in developed countries.
Knowing why people are physically inactive is therefore of key importance in developing strategies to reduce these major diseases. Active commuting is a cheap and easy way to incorporate regular physical activity into daily life. The purpose of this study was to identify what individual and environmental factors are associated with active commuting.

Methods Cross-sectional data were obtained from residents (age 25-75) of 87 neighbourhoods in the city of Eindhoven, the Netherlands, who participated in the GLOBE study in 2004 $(\mathrm{N}=2474)$. We used multilevel logistic regression models to analyse associations of perceived neighbourhood factors (attractiveness, safety) and individual cognitions regarding physical activity (attitude, self-efficacy, intention, social influence) with active commuting (yes/no).

Results People with a positive attitude, a high self-efficacy, a strong intention to be physically active, and positive social support, were more likely to engage in active commuting. People reporting to sometimes feel unsafe in their neighbourhood were less likely to engage in active commuting compared to those who perceived their neighbourhood as safe. Perceived neighbourhood attractiveness was not significantly associated with active commuting.

Conclusion When stimulating active commuting, it is important to focus on changing individual cognitions towards physical activity. Environmental changes, for example, improving neighbourhood safety may also increase active commuting. Future studies should identify more potentially relevant neighbourhood factors.

\section{P1-395 INEQUALITIES OF IODINE INTAKE IN PREGNANT WOMEN AFTER SALT IODISATION: LOW \& HIGH QUANTILES AND CORRELATED FACTORS}

doi:10.1136/jech.2011.142976f.86

A Beiranvand, ${ }^{*}$ S Salarilak, J Nouroozzadeh, H Khalkhali, M Aghasi, R Rostami. Urmia University of medical science, Urmia, West Azarbaijan, Iran

Introduction Iodine is scarcely found in food products of many regions through the world. Scientific findings have suggested that providing adequate iodine during pregnancy prevents many adverse effects of iodine deficiency (ID) in newborn babies. Therefore, Pregnant women are a special group in this context.

Material \& method 1078 pregnant women at their first prenatal care from five districts of Urmia City, north west of Iran, were interviewed. Of these, 490 were qualified and followed until delivery. Urine and sera samples were collected at two stages (1st \& 3rd trimester). Data of demographic features and iodised salt accessibility were gathered through a questionnaire.

Results Mean age (SD) of studied people was 25 (5.4) years. All households had proper access to iodised salt. Median of Urinary iodine excretion (UIE) was $73.5 \mu \mathrm{g} / 1$ as a whole at 1 st trimester. This raised to $114 \mu \mathrm{g} / \mathrm{l}$ at 3rd trimester. According to WHO criterion, $87 \%$ and $70 \%$ iodine deficiency (ID) was detected at 1 st \& 3rd trimester, respectively. Quantile regression models for 1st trimester showed educational status, way of using iodised salt, and geographical situation as determinant factors in first\&third quartile of UIE. The same results were observed at $3 \mathrm{rd}$ trimester.

Conclusion Nearly 20 years after salt iodisation program in Iran, there are pockets of iodine deficiency even in a close vicinity. Education and knowledge about using iodised salt are major determinant factors in iodine intake of pregnant women. It seems that more practice on this context is needed to assure adequate access to iodine generally and for pregnant women specifically. 